H. Tango

Nagoya Math. J.

Vol. 48 (1972), 73-89

\title{
ON THE BEHAVIOR OF EXTENSIONS OF VECTOR BUNDLES UNDER THE FROBENIUS MAP
}

\author{
HIROSHI TANGO
}

\section{Introduction.}

Let $k$ be an algebraically closed field of characteristic $p>0$, and let $X$ be a curve defined over $k$. The aim of this paper is to study the behavior of the Frobenius map $F^{*}: H^{1}(X, E) \rightarrow H^{1}\left(X, F^{*} E\right)$ for a vector bundle $E$.

Our main result is the following.

THEOREM 15. Let $X$ be a curve of genus $g>0$. Let $n(X)$ be the integer defined by

$$
\begin{array}{r}
\boldsymbol{n}(X)=\max \left\{\sum_{x \in X}\left[\frac{v_{x}(\mathrm{~d} f)}{p}\right] ; f \text { runs over all rational functions on } X\right. \\
\text { with } \mathrm{d} f \neq 0\} .
\end{array}
$$

Then

(i) for any line bundle $L$ such that $\operatorname{deg} L>\boldsymbol{n}(X)$, the Frobenius map $F^{*}: H^{1}(X, \check{L}) \rightarrow H^{1}\left(X, F^{*} \check{L}\right)$ is injective.

(ii) if $\boldsymbol{n}(X)>0$, then there exists a line bundle $M$ of degree $\boldsymbol{n}(X)$ such that the Frobenius map $F^{*}: H^{1}(X, \check{M}) \rightarrow H^{1}\left(X, F^{*} \check{M}\right)$ is not injective. (where $\check{L}$ is the dual line bundle of $L$ )

This main result leads us to a counter example to a question posed by R. Hartshorne:

Question. Assume the Hasse-Witt matrix of $X$ is non-singular. Is the Frobenius map $F^{*}: H^{1}(X, \check{L}) \rightarrow H^{1}\left(X, F^{*} \check{L}\right)$ injective for any ample line bundle $L$ ?

I wish to thank Professor Hideyasu Sumihiro for his advice and Received September 4, 1971.

Revised May 19, 1972. 
encouragement, and Professors Masayoshi Nagata and Masaki Maruyama for their valuable conversations.

\section{Notations.}

Throughout this paper, we mean by a variety (resp. curve) an irreducible complete non-singular variety (resp. curve) defined over an algebraically closed field of characteristic $p>0$. We denote by $\mathcal{O}_{X}$ the structure sheaf of $X$, by $K=K(X)$ the field of rational functions on $X$ and by $\Omega_{X}^{i}$ the sheaf of germs of regular differential $i$-forms.

We use the words vector bundle and locally free sheaf interchangeably. For any vector bundle $E$ of rank $n$ on a curve, there exists a series of subbundles of $E$

$$
0=E_{0} \subset E_{1} \subset E_{2} \subset, \cdots, \subset E_{n}=E
$$

where $L_{i}=E_{i} / E_{i-1}$ is a line bundle (cf. Atiyah [1]) $\left(L_{1}, L_{2}, \cdots, L_{n}\right)$ will be called a splitting of $E$. A line subbundle $L$ of $E$ will be called a maximal line subbundle of $E$, if $L$ satisfies the following condition: for any line subbundle $M$ of $E$, $\operatorname{deg} L \geqq \operatorname{deg} M$.

A splitting $\left(L_{1}, L_{2}, \cdots, L_{n}\right)$ will be called a maximal splitting of $E$, if it satisfies the following conditions:

(i) $L_{1}$ is a maximal line subbundle of $E$,

(ii) $\left(L_{2}, L_{3}, \cdots, L_{n}\right)$ is a maximal splitting of $E / L_{1}$.

We denote by $\check{E}$ the dual vector bundle of $E$ and denote by $h^{i}(E)$ the dimension of the $k$-vector space $H^{i}(X, E)$.

1. Let $X$ be a variety of $\operatorname{dim} n$. Let $F: X \rightarrow X$ be the Frobenius morphism. (cf. [4]). The natural derivation $\mathrm{d}: \mathcal{O}_{X} \rightarrow \Omega_{X}^{1}$ gives rise to a $k$-linear map d: $\Omega_{X}^{i} \rightarrow \Omega_{X}^{i+1}$ for each $i$, which induce $a \mathcal{O}_{X}$-homomorphism $F_{*} \mathrm{~d}: F_{*} \Omega_{X}^{i} \rightarrow F_{*} \Omega_{X}^{i+1}$ for each $i$. We denote by $\mathscr{Z}_{X}^{i}$ (resp. $\mathscr{B}_{X}^{i+1}$ ) the kernel (resp. image) of $F_{*} \mathrm{~d}: F_{*} \Omega_{X}^{i} \rightarrow F_{*} \Omega_{X}^{i+1}$. Let $x$ be a point of $X$ and let $u_{1}, u_{2}, \cdots, u_{n}$ be local parameters of $X$ at $x$. Then we have the following Propositions, due to Cartier (cf. [10]).

Proposition 1. $\quad \mathscr{Z}_{X X}^{i}=\mathscr{B}_{X, x}^{i} \oplus\left(\oplus \mathcal{O}_{X, x}^{P}\left(u_{j_{1}}, u_{j_{2}}, \cdots, u_{j_{i}}\right)^{p-1} \mathrm{~d} u_{j_{1}} \wedge \mathrm{d} u_{j_{2}}\right.$ $\wedge \cdots \wedge \mathrm{d} u_{j_{i}}$ ) where $\mathcal{O}_{X, x}^{P}=\left\{f^{p} ; f \in \mathcal{O}_{X, x}\right\}, \mathscr{Z}_{X, x}^{i}$ is an $\mathcal{O}_{X, x}$-module through the $p$-th power map. 
Proposition 2. There are $\mathcal{O}_{X}$-homomorphisms $C: \mathscr{Z}_{X}^{i} \rightarrow \Omega_{X}^{i}$, called the Cartier operator, with the following properties.

(i ) $C\left(\omega_{1}+\omega_{2}\right)=C\left(\omega_{1}\right)+C\left(\omega_{2}\right)$

(ii) $C\left(f^{p} \omega\right)=f C(\omega)$

(iii) $\quad C(\omega)=0 \quad$ if $\omega \in \mathscr{B}_{X, x}^{i}$

(iv) $C\left(\left(f_{1} f_{2} \cdots f_{i}\right)^{p-1} \mathrm{~d} f_{1} \wedge \mathrm{d} f_{2} \wedge \cdots \wedge \mathrm{d} f_{i}=\mathrm{d} f_{1} \wedge \mathrm{d} f_{2} \wedge \cdots \wedge \mathrm{d} f_{i}\right.$ where $\omega_{1}, \omega_{2}, \omega \in \mathscr{Z}_{X, x}^{i}$ and $f, f_{1}, f_{2}, \cdots, f_{i} \in \mathcal{O}_{X, x}$.

Proposition 3. The following sequence of $\mathcal{O}_{X}$-Modules are exact.

(i) $0 \longrightarrow \mathscr{Z}_{X}^{i} \longrightarrow F_{*} \Omega_{X}^{i} \stackrel{F_{*} \mathrm{~d}}{\longrightarrow} \mathscr{B}_{X}^{i+1} \longrightarrow 0$

(ii) $0 \longrightarrow \mathcal{O}_{X} \stackrel{F^{\prime}}{\longrightarrow} F_{*} \mathcal{O}_{X} \stackrel{F_{* d}}{\longrightarrow} \mathscr{B}_{X}^{1} \longrightarrow 0$

(iii) $0 \longrightarrow \mathscr{B}_{X}^{i} \longrightarrow \mathscr{Z}_{X}^{i} \stackrel{C}{\longrightarrow} \Omega_{X}^{i} \longrightarrow 0$

Since the Frobenius morphism $F$ is affine, the canonical $p$-linear map $\alpha: H^{i}\left(X, F_{*} \mathscr{F}\right) \rightarrow H^{i}(X, \mathscr{F})$ is bijective, for any coherent sheaf $\mathscr{F}$ on $X$ and for any integer $i$, (cf. [3] III. 1. 3. 3.). Since $\mathscr{Z}_{X}^{n}=F_{*} \Omega_{X}^{n}, \operatorname{dim} H^{n}\left(X, \mathscr{Z}_{X}^{n}\right)$ $=\operatorname{dim} H^{n}\left(X, \Omega_{X}^{n}\right)=1$ and the Cartier operator $C^{*}: H^{n}\left(X, \mathscr{Z}_{X}^{n}\right) \rightarrow H^{n}\left(X, \Omega_{X}^{n}\right)$ is surjective, so we have that $C^{*}$ is bijective. Let $E$ be a vector bundle on $X$. Then there exists a natural map $\psi: E \otimes \check{E} \otimes \Omega_{X}^{n} \rightarrow \Omega_{X}^{n}$ and the cup product

$$
U: H^{i}(X, E) \times H^{n-i}\left(X, \check{E} \otimes \Omega_{X}^{n}\right) \longrightarrow H^{n}\left(X, E \otimes \check{E} \otimes \Omega_{X}^{n}\right) .
$$

The composition map

$$
H^{i}(X, E) \times H^{n-i}\left(X, \check{E} \otimes \Omega_{X}^{n}\right) \longrightarrow H^{n}\left(X, E \otimes \check{E} \otimes \Omega_{X}^{n}\right) \longrightarrow H^{n}\left(X, \Omega_{X}^{n}\right) \approx k .
$$

gives the Serre duality between $H^{i}(X, E)$ and $H^{n-i}\left(X, E \otimes \Omega_{X}^{n}\right)$.

The following is well known (e.g. for curves Serre [9]).

Proposition 4. Let $E$ be a vector bundle on $X$. Then the following two k-linear maps are dual to each other.

(i) $F^{* *}(i, E): H^{i}(X, E) \longrightarrow H^{i}\left(X, E \otimes F_{*} \mathcal{O}_{X}\right)$

(ii) $C^{*}(n-i, \check{E}): H^{n-i}\left(X, \check{E} \otimes \mathscr{Z}_{X}^{n}\right) \longrightarrow H^{n-i}\left(X, \check{E} \otimes \Omega_{X}^{n}\right)$.

In particular, we have dim Image $F^{* *}(i, E)=\operatorname{dim} \operatorname{Image} C^{*}(n-i, \check{E})$.

For the sake of completeness we include a proof: 
$H^{i}(X, E) \times H^{n-i}\left(X, \check{E} \otimes \Omega_{X}^{n}\right) \stackrel{U}{\longrightarrow} H^{n}\left(X, E \otimes \check{E} \otimes \Omega_{X}^{n}\right) \stackrel{\psi^{*}}{\longrightarrow} H^{n}\left(X, \Omega_{X}^{n}\right) \approx k$ $\uparrow i d \times C *(n-i, \check{E}) \quad \quad \quad \uparrow C *(n, E \otimes \check{E}) \quad \uparrow C *\left(n, \mathcal{O}_{X}\right)$ $H^{i}(X, E) \times H^{n-i}\left(X, \check{E} \otimes \mathscr{Z}_{X}^{n}\right) \stackrel{U}{\longrightarrow} H^{n}\left(X, E \otimes \check{E} \otimes \mathscr{Z}_{X}^{n}\right) \stackrel{\psi^{*}}{\longrightarrow} H^{n}\left(X, \mathscr{Z}_{X}^{n}\right)$ $\downarrow F^{\prime *}(i, E) \times i d$

$H^{i}\left(X, E \otimes F_{*} \mathcal{O}\right) \times H^{n-i}\left(X, \check{E} \otimes \mathscr{Z}_{X}^{n}\right)$<smiles>[Y][Y]</smiles>
$H^{i}\left(X, F^{*} E\right) \times H^{n-i}\left(X, F^{*} \check{E} \otimes \Omega_{X}^{n}\right) \stackrel{U}{\longrightarrow} H^{n}\left(X, F^{*} E \otimes F^{*} \check{E} \otimes \Omega_{X}^{n} \stackrel{\psi^{*}}{\longrightarrow} H^{n}\left(X, \Omega_{X}^{n}\right)\right.$

Giving the duality between $H^{i}\left(X, E \otimes F_{*} \mathcal{O}_{X}\right)$ and $H^{n-i}\left(X, \check{E} \otimes \mathscr{Z}_{X}^{n}\right)$ by the composition map $C^{*}\left(n, \mathcal{O}_{X}\right) \circ \alpha^{-1} \circ \psi^{*} \circ U \circ(\alpha \times a)$, we have the duality between $F^{\prime *}(i, E)$ and $C^{*}(n-i, E)$.

2. Let $E$ be a vector bundle on $X$. We denote by $F^{*}(i, E)$, the composition map $\alpha \circ F^{\prime *}(i, E): H^{i}(X, E) \rightarrow H^{i}\left(X, F^{*} E\right)$.

THEOREM 5. Let $X$ be a curve and let $E$ be a vector bundle on $X$. Then

(i) $\operatorname{dim}$ Cokernel $F^{*}(1, E)=h^{0}\left(\check{E} \otimes \mathscr{B}_{X}^{1}\right)$

(ii) $\operatorname{dim}$ Kernel $F^{*}(1, \check{E})=h_{0}\left(\check{E} \otimes \mathscr{B}_{X}^{1}\right)-\left(h^{0}\left(F^{*} \check{E}\right)-h^{0}(\check{E})\right)$ $\leqq h^{0}\left(\check{E} \otimes \mathscr{B}_{X}^{1}\right)$

Proof. By virtue of Proposition 3, we have the following exact sequences,

$$
\begin{aligned}
& 0 \longrightarrow \check{E} \otimes \mathscr{B}_{X}^{1} \longrightarrow \check{E} \otimes \mathscr{Z}_{X}^{1} \stackrel{C}{\longrightarrow} \check{E} \otimes \Omega_{X}^{1} \longrightarrow 0 \\
& 0 \longrightarrow \check{E} \longrightarrow F_{*} \mathcal{O}_{X} \longrightarrow \check{E} \otimes \mathscr{B}_{X}^{1} \longrightarrow 0
\end{aligned}
$$

and hence following cohomology exact sequences

$$
\begin{aligned}
& 0 \longrightarrow H^{0}\left(X, \check{E} \otimes \mathscr{B}_{X}^{1}\right) \longrightarrow H^{0}\left(X, \check{E} \otimes \mathscr{Z}_{X}^{1}\right) \stackrel{C *(0, \check{E})}{\longrightarrow} H^{0}\left(X, \check{E} \otimes \Omega_{X}^{1}\right) \\
& 0 \longrightarrow H^{0}(X, \check{E}) \quad \longrightarrow H^{0}\left(X, \check{E} \otimes F_{*} \mathcal{O}_{X}\right) \longrightarrow H^{0}\left(X, \check{E} \otimes \mathscr{B}_{X}^{1}\right) \\
& \longrightarrow H^{1}(X, \check{E}) \stackrel{. F^{*} *(1, \check{E})}{\longrightarrow} H^{1}\left(X, \check{E} \otimes F_{*} \mathcal{O}_{X}\right)
\end{aligned}
$$

Hence we have

$$
\begin{aligned}
\operatorname{dim} & \text { Cokernel } F^{*}(1, E)=\operatorname{dim} \text { Cokernel } F^{*}(1, E) \\
& =h^{1}\left(E \otimes F_{*} \mathcal{O}_{X}\right)-\operatorname{dim} \operatorname{Image} F^{* *}(1, E) \\
& =h^{1}\left(F^{*} E\right)-\operatorname{dim} \text { Image } C^{*}\left(0, \check{E}^{\prime}\right) \quad(\text { by virtue of Proposition } 4) \\
& =h^{1}\left(F^{*} E\right)-\left(h^{0}\left(\check{E} \otimes F_{*} \Omega_{X}^{1}\right)-h^{0}\left(\check{E} \otimes \mathscr{B}_{X}^{1}\right)\right) \\
& =h^{0}\left(\check{E} \otimes \mathscr{B}_{X}^{1}\right)
\end{aligned}
$$


And we have

$$
\begin{aligned}
\operatorname{dim} \operatorname{Kernel} & F^{*}(1, \check{E})=\operatorname{dim} \operatorname{Kernel} F^{\prime *}(1, \check{E}) \\
& =h^{0}\left(\check{E} \otimes \mathscr{B}_{X}^{1}\right)-h^{0}\left(\check{E} \otimes F_{*} \mathcal{O}_{X}\right)+h^{0}(\check{E}) \\
& =h^{0}\left(\check{E} \otimes \mathscr{B}_{X}^{1}\right)-\left(h^{0}\left(F^{*} \check{E}\right)-h^{0}(\check{E})\right) \\
& \leqq h^{0}\left(\check{E} \otimes \mathscr{B}_{X}^{1}\right)
\end{aligned}
$$

CoRollary 6. Let $X$ be a curve and let $E$ be a vector bundle. Assume that the Frobenius map $F^{*}(1, E)$ is surjective, then $F^{*}(1, \check{E})$ is injective and $h^{0}\left(F^{*} \check{E}\right)=h^{0}(\check{E})$.

As a corollary of this Theorem 5, we have the following Theorem of Oda:

THEOREM 7. (T. Oda). Let $X$ be an elliptic curve and let $E$ be an indecomposable vector bundle of rank $r$ and of degree $d$. Then we have the following results.

(i) When the Hasse-Witt matrix of $X$ is not zero (i.e., $F^{*}\left(1, \mathcal{O}_{X}\right)$ is injective), the Frobenius $\operatorname{map} F^{*}(1, E)$ is injective.

(ii) When the Hasse-Witt matrix of $X$ is zero (i.e., $F^{*}\left(1, \mathcal{O}_{X}\right)$ is the zero map), the Frobenius map $F^{*}(1, E)$ is not injective (and in fact the zero map) if and only if $r<p, d=0$ and $E$ has a non-zero section (i.e., in Atiyah's notation $E=F_{r}$ with $r<p$ ).

CoRollary 8. (Corollary of the proof of Theorem 7) (cf. [1] p. 451) Let $X$ be an elliptic curve.

(i) When the Hasse-Witt matrix of $X$ is not zero, then $\mathscr{B}_{X}^{1} \approx L_{1} \oplus L_{2} \oplus \ldots \oplus L_{p-1}$ where $\left\{\mathcal{O}_{X}, L_{1}, L_{2}, \cdots, L_{p-1}\right\}=\left\{L ;\right.$ line bundles with $\left.L^{\otimes P} \approx \mathcal{O}_{X}\right\}$

(ii) When the Hasse-Witt matrix of $X$ is zero, then $\mathscr{B}_{X}^{1} \approx F_{p-1}$.

(iii) $F^{*} F_{*} \mathcal{O}_{X} \approx \stackrel{p}{\oplus} \mathcal{O}_{X}$

Proof. Let $E$ be an indecomposable vector bundle of rank $r$ and of degree $d$. We use the following results of Atiyah (cf. [1]).

$$
\begin{aligned}
& h^{0}(E)=d \text { and } h^{1}(E)=0 \text { when } d \text { is positive } \\
& h^{0}(E)=0 \text { and } h^{1}(E)=-d \text { when } d \text { is negative. } \\
& h^{0}(E)=h^{1}(E)=0 \text { when } d=0 \text { and } E \neq F_{r} . \\
& h^{0}(E)=h^{1}(E)=1 \text { when } E \approx F_{r} .
\end{aligned}
$$


When $d=0$, there is a line bundle of degree 0 with $E \approx L \otimes F_{r}$. It is easy to see that $\mathscr{B}_{X}^{1}$ is a vector bundle of rank $p-1$. Let $\mathscr{B}_{X}^{1} \approx E_{1} \oplus E_{2} \oplus \cdots \oplus E_{s}$ be the decomposition of $\mathscr{B}_{X}^{1}$ into indecomposable factors. Let $r_{i}$ be the rank of $E_{i}$ and let $d_{i}$ be the degree of $E_{i}$. Then we have $\sum \mathrm{d}_{i}=\operatorname{deg} \mathscr{B}_{X}^{1}=\chi\left(\mathscr{B}_{X}^{1}\right)=\chi\left(F_{*} \mathcal{O}_{X}\right)-\chi\left(\mathcal{O}_{X}\right)=0$. Let $L$ be a non trivial line bundle of degree 0 , then $h^{0}\left(L \otimes \mathscr{B}_{X}^{1}\right) \neq 0$ (in fact equal to 1) if and only if $L^{\otimes P} \approx \mathcal{O}_{X}$ by virtue of following exact sequence.

$$
0=H^{0}(X, L) \longrightarrow H^{0}\left(X, L \otimes F_{*} \mathcal{O}_{X}\right) \longrightarrow H^{0}\left(X, L \otimes \mathscr{B}_{X}^{1}\right) \longrightarrow H^{1}(X, L)=0 \text {. }
$$

This shows that $d_{i} \leqq 0$ for all $i$ and so $d_{i}=0$ for all $i$. Let $L_{i}$ be the line bundle with $E_{i} \approx L_{i} \otimes F_{r_{i}}$, then $L_{i}^{\otimes P} \approx \mathcal{O}_{X}$. By virtue of Lemma 13, we have the following results. When $h^{0}\left(\mathscr{B}_{X}^{1}\right)=1$, then $s=1, r_{1}=p-1$ and $L_{1} \approx \mathcal{O}_{X}$. And when $h^{0}\left(\mathscr{B}_{X}^{1}\right)=0$, then $s=p-1, r_{i}=1$ and $\left\{\mathcal{O}_{X}, L_{1}, L_{2}, \cdots, L_{p_{-1}}\right\}=\left\{L\right.$; line bundles with $\left.L^{\otimes P} \approx \mathcal{O}_{X}\right\}$. Let $E$ be an indecomposable vector bundle of rank $r$ and of degree $d$. If $d>0$, then $h^{1}(E)=0$. If $d<0$, then $h^{0}(E \otimes L)=0$ for all line bundle $L$ of degree 0 , and so $h^{0}\left(E \otimes \mathscr{B}_{X}^{1}\right)=0$. Thus the Frobenius map $F^{*}(1, E)$ is injective when $d \neq 0$. When $d=0$ and $E \neq F_{r}$ then $h^{1}(E)=0$ and the Frobenius map $F^{*}(1, E)$ is injective. When $E=F_{r}$ and the Hasse-Witt matrix of $X$ is not zero, then $h^{0}\left(E \otimes \mathscr{B}_{X}^{1}\right)=0$ and the Frobenius map $F^{*}(1, E)$ is injective. When $E=F_{r}$ and the Hasse-Witt matrix of $X$ is zero, then we have the following results by induction on $r . h^{0}\left(F^{*} F_{r}\right)=\min \{p, r\}$, $F^{*} F_{r} \approx \oplus^{r} \mathcal{O}_{X}$ for all $r$ with $r \leqq p$ and $F^{*}\left(1, F_{r}\right)$ is the zero map if and only if $r \leqq p-1$.

$r=1$. It is obvious.

$p \geqq r>1$. We have the following exact sequence

$$
0 \longrightarrow F_{r-1} \longrightarrow F_{r} \longrightarrow \mathcal{O}_{X} \longrightarrow 0 \text {. }
$$

Hence we have $F^{*} F_{r} \approx \stackrel{r}{\oplus} \mathcal{O}_{X}$ and $h^{0}\left(F^{*} F_{r}\right)=r$ by the induction assumption. But we have $h^{0}\left(F_{r} \otimes \mathscr{B}_{X}^{1}\right)=h^{0}\left(F_{r} \otimes F_{p-1}\right)=\min \{r, p-1\}$ (for all $r$, cf. [1] Lemma 17). Hence we have $h^{0}\left(F_{r} \otimes \mathscr{B}_{X}^{1}\right)-h^{0}\left(F^{*} F_{r}\right)+h^{0}\left(F_{r}\right)=1$, if $r<p$. This shows that when $r<p$ the Frobenius map $F^{*}\left(1, F_{r}\right)$ is the zero map.

$p \leqq r . \quad F_{r}$ has $F_{p}$ as a subbundle and so $h^{0}\left(F^{*} F_{r}\right) \geqq p$ by the induction assumption. Hence we have

$0 \leqq h\left(F_{r} \otimes \mathscr{B}_{X}^{1}\right)-h^{0}\left(F^{*} F_{r}\right)+h^{0}\left(F_{r}\right) \leqq 0$. This shows that $h_{0}\left(F^{*} F_{r}\right)=p$ and the Frobenius map $F^{*}\left(1, F_{r}\right)$ is injective. 
3. Let $X$ be a curve. For any divisor $D$ on $X$, we denote by $\mathcal{O}(D)$ the line bundle associated with $D$.

DEFINITION 9. For any function $f \in K$, we denote by $\boldsymbol{n}(f)$ the integer (or infinity) $\sum_{x \in X}\left[v_{x}(\mathrm{~d} f) / p\right]$ where [ ] is the Gauss symbol, and $v_{x}$ is the valuation associated with $x$.

LEMMA 10. Let $g$ be the genus of the curve $X$. Then

(i) $\boldsymbol{n}(f)=\infty$ if and only if $f \in K^{p}$,

(ii) $\boldsymbol{n}(f) \leqq[2(g-1) / p]$, if $\boldsymbol{n}(f)<\infty$.

Proof. $\mathrm{d} f=0$ if and only if $f \in K^{p}$, and if $\mathrm{d} f=0$, then $n(f)=\infty$. If $\mathrm{d} f \neq 0$, the divisor $D=\sum_{x \in X} v_{x}(\mathrm{~d} f) x$ is a canonical divisor, and so the degree of $D$ is $2(g-1)$. Therefore we have

$$
n(f) \leqq\left[\frac{2(g-1)}{p}\right] \text {. }
$$

DEFINITION 11. We define $\boldsymbol{n}(X)$ by the following formula

$$
\boldsymbol{n}(X)=\max \left\{\boldsymbol{n}(f) ; f \in K \text { and } f \notin K^{p}\right\} .
$$

Note that $n(X) \leqq[2(g-1) / p]$, by virtue of Lemma 10 .

LEMMA 12. Let $D$ be a divisor on $X$, Then we have

$$
H^{0}\left(X, \mathcal{O}(-D) \otimes \mathscr{B}_{X}^{1}\right) \cong\{\mathrm{d} f ; f \in K \text { and }(\mathrm{d} f)>p D\} \text {. }
$$

Proof. By virtue of Proposition 3, we have the following exact sequence.

$$
0 \longrightarrow \mathcal{O}(-D) \otimes \mathscr{B}_{X}^{1} \longrightarrow \mathcal{O}(-D) \otimes \mathscr{Z}_{X}^{1} \stackrel{C}{\longrightarrow} \mathcal{O}(-D) \otimes \Omega_{X}^{1} \longrightarrow 0
$$

Hence, we have the following cohomology exact sequence.

$$
\begin{aligned}
0 \longrightarrow H^{0}\left(X, \mathcal{O}(-D) \otimes \mathscr{B}_{X}^{1}\right) \longrightarrow & H^{0}\left(X, \mathcal{O}(-D) \otimes \mathscr{Z}_{X}^{1}\right) \stackrel{C *}{\longrightarrow} H^{0}\left(X, \mathcal{O}(-D) \otimes \Omega_{X}^{1}\right) \\
& H^{0}\left(X, \mathcal{O}(-p D) \otimes \Omega_{X}^{1}\right)
\end{aligned}
$$

Since, $H^{0}\left(X, \mathcal{O}(-p D) \otimes \Omega_{X}^{1}\right)=\left\{\omega \in \Omega^{1}(K / k) ;(\omega)>p D\right\}$. The assersion is obvious by virtue of Proposition 3.

Remark: By Lemma 12, it is easy to see that $n(X)$ coincides with the degree of a maximal line subbundle of $\mathscr{B}_{X}^{1}$.

LEMMA 13. Let $G$ be the group of linear equiualence classes of 
divisors on $X$, and let $G_{p}$ be the subgroup of elements $\bar{D} \in G$ such that $p \bar{D}=0$. Then $G_{p}$ is a finite group of order $p^{\sigma}$, where $\sigma$ is the rank of the Hasse-Witt matrix of $X$.

Proof. See Serre [9] Proposition $10 \S 2$.

Proposition 14. Let $X$ be a curve of genus $g>0$. Then $n(X) \geqq 0$.

Proof. When the Hasse-Witt matrix of $X$ is not zero. $G_{p} \neq 0$, by virtue of Lemma 13 . So there exists a non-zero element $\bar{D} \in G$ such that $p \bar{D}=0$. Therefore, there exists a rational function $f$ such that $f \notin K^{p}$ and $(f)=p D$. Hence $(\mathrm{d} f)>p D$. Thus $n(X) \geqq \operatorname{deg} D=0$.

When the Hasse-Witt matrix of $X$ is zero, i.e., $F^{*}\left(1, \mathcal{O}_{X}\right)$ is the zero map. We have

$$
0 \longrightarrow H^{0}\left(X, \mathcal{O}_{X}\right) \longrightarrow H^{0}\left(X, F^{*} \mathcal{O}_{X}\right) \longrightarrow H^{0}\left(X, \mathscr{B}_{X}^{1}\right) \longrightarrow H^{1}\left(X, \mathcal{O}_{X}\right) \longrightarrow 0
$$

and hence we have $H^{0}\left(X, \mathscr{B}_{X}^{1}\right) \approx H^{1}\left(X, \mathcal{O}_{X}\right) \neq 0$. Therefore $n(X) \geqq 0$, by virtue of Remark.

THEOREM 15. Let $X$ be a curve of genus $g>0$. Then

(i) for any line bundle $L$ such that $\operatorname{deg} L>n(X)$, the Frobenius map $F^{*}(1, \check{L}): H^{1}(X, \check{L}) \rightarrow H^{1}\left(X, F^{*} \check{L}\right)$ is injective.

(ii) if $\boldsymbol{n}(X)>0$, then there exists a line bundle $M$ of degree $n(X)$ such that the Frobenius map $F^{*}(1, \check{M})$ is not injective.

Proof. Let $\operatorname{deg} L>\boldsymbol{n}(X)$. Then $H^{0}\left(X, \check{L} \otimes \mathscr{B}_{X}^{1}\right)=0$ by virtue of Remark. Therefore the Frobenius map $F^{*}(1, \check{L})$ is injective by virtue Theorem 5.

(ii) $n(X)>0$. There exists a line bundle $M$ of degree $n(X)>0$, with $H^{0}\left(X, \check{M} \otimes \mathscr{B}_{X}^{1}\right) \neq 0$. Since $h^{0}\left(F^{*}(\check{M})\right)=0$, the Frobenius map $F^{*}(1, \check{M})$ is not injective by virtue of Theorem 5 .

The following Proposition gives the relation between the number $\boldsymbol{n}(X)$ and the rank of the Hasse-Witt matrix.

Proposition 16. Let $X$ be a curve of genus $g>0$, and let $\boldsymbol{h}(X)$ be the rank of the Hasse-Witt matrix of $x$. Then we have

$$
g-h(X) \leqq(p-1)(n(X)+1)
$$

Proof. Let $D$ be an effective divisor of degree $d>0$, such that the 
Frobenius map $F^{*}(1, \mathcal{O}(-D))$ is injective. Then we have the following exact commutative diagram.

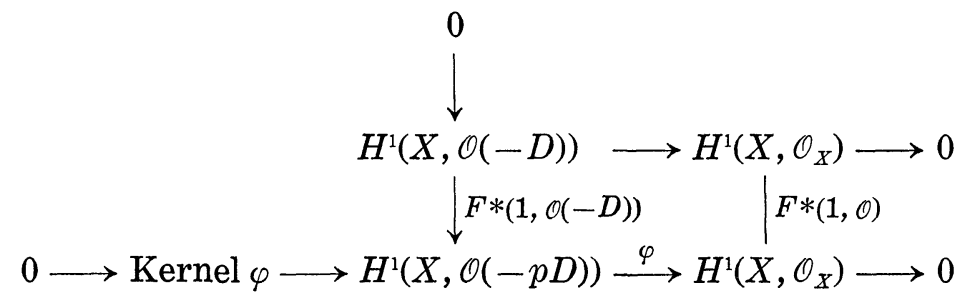

And we have

$\operatorname{dim}$ Image $\varphi \circ F^{*}(1, \mathcal{O}(-D)) \geqq h^{1}(\mathcal{O}(-D))-\operatorname{dim} \operatorname{Kernel} \varphi=g+d-p d$.

Hence we have $h(X) \geqq g+d-p d$, i.e., $g-h(X) \leqq(p-1) d$. Since, for any effective divisor $D$ of degree $n(X)+1$, the Frobenius map $F^{*}(1, \mathcal{O}(-D))$ is injective, we have

$$
g-\boldsymbol{h}(X) \leqq(p-1)(\boldsymbol{n}(X)+1) .
$$

4. In this section we shall extend Theorem 15 from line bundles to indecomposable vector bundles of arbitrary rank.

Proposition 17. Let $X$ be a curve of genus $g>0$. Then for any $r$, there exists an indecomposable vector bundle which has a splitting

$$
\left(\Omega_{X}^{1 \otimes(r-1)}, \Omega_{X}^{1 \otimes(r-2)}, \cdots, \Omega_{X}^{1}, \mathcal{O}_{X}\right) .
$$

In order to prove Proposition 17, we need the following Lemmas.

LEMMA 18. Let $E$ and $E^{\prime}$ be vector bundle on $X$, and $\operatorname{let}\left(L_{1}, L_{2}, \cdots, L_{r}\right)$ be a splitting of $E$, and suppose that $\varphi: E \rightarrow E^{\prime}$ is a generically surjective morphism. Then there exists a splitting $\left(L_{1}^{\prime}, L_{2}^{\prime}, \cdots, L_{s}^{\prime}\right)$ of $E^{\prime}$ which satisfies the following condition; There exists a sequence $1 \leqq i_{1}<i_{2}<$, $\cdots,<i_{s}$ such that Hom $\left(L_{i_{j}}, L_{j}^{\prime}\right) \neq 0$ for all $j$, in particular $\operatorname{deg} L_{i_{j}} \leqq \operatorname{deg} L_{j}^{\prime}$.

Proof of Lemma 18. It is easy.

Lemma 19. Let $X$ be a curve and let $E^{\prime}$ be an indecomposable vector bundle which has a splitting $\left(L_{1}, L_{2}, \ldots, L_{r}\right)$. Let $L$ be a line bundle such that $\operatorname{deg} L<\operatorname{deg} L_{j}$ for all $j$. If an exact sequence $0 \longrightarrow E^{\prime} \stackrel{\alpha}{\longrightarrow} E \stackrel{\varphi}{\longrightarrow} L \longrightarrow 0$ does not split, then $E$ is indecomposable.

Proof of Lemma 19. Tensoring the sequence with $\check{L}$ we may assume 
that $L=\mathcal{O}_{X}$ and $\operatorname{deg} L_{j}>0$ for all $j$. Suppose $E$ is decomposable. Let $E=E_{1} \oplus E_{2}$ and let $\psi_{i}$ be the injection $E_{i} \rightarrow E \quad(i=1,2)$. We may assume that $\varphi \circ \psi_{1} \neq 0$. By virtue of Lemma 18, there exists a splitting $\left(L_{1}^{\prime}, L_{2}^{\prime}, \cdots, L_{r_{1}}^{\prime}\right)$ of $E_{1}$ such that $\operatorname{deg} L_{i}^{\prime} \geqq 0$ for all $i$. Therefore $\varphi \circ \psi$ is surjective. And we have the following exact commutative diagram.

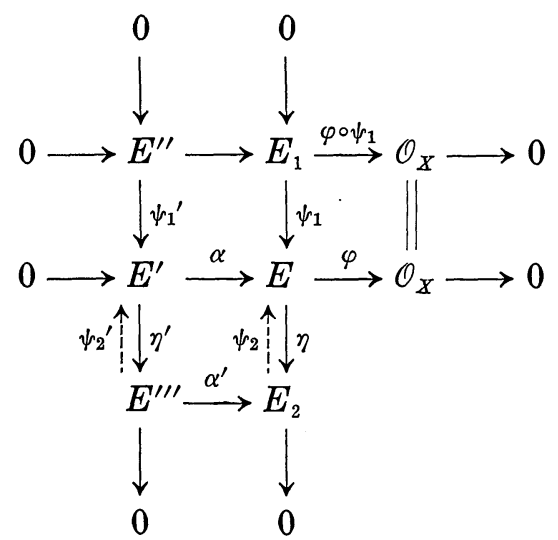

where $E^{\prime \prime}$ is the kernel of $\varphi \circ \psi_{1}, \psi_{1}^{\prime}$ is the injection induced by $\psi_{1}, E^{\prime \prime \prime}$ is the cokernel of $\psi_{1}^{\prime}$ and $\alpha^{\prime}$ is the homomorphism induced by $\alpha$. By virtue of Snake Lemma, the map $\alpha^{\prime}$ is an isomorphism. Since $\operatorname{deg} L_{i}>0$ for all $j$, the composition map $\varphi \circ \psi_{2} \circ \alpha^{\prime} \circ \eta^{\prime}=0$, by virtue of Lemma 18, since $\eta$ is a surjection, $\varphi \circ \psi_{2} \circ \alpha^{\prime}=0$. Hence, the exists a map $\psi_{2}^{\prime}: E^{\prime \prime \prime} \rightarrow E^{\prime}$ such that $\alpha \circ \psi_{1}^{\prime}=\psi_{2} \circ \alpha^{\prime}$. It is easy to show that $\eta^{\prime} \circ \psi_{2}^{\prime}=$ identity. Therefore $E^{\prime}=E^{\prime \prime} \oplus E^{\prime \prime \prime} \cdot E^{\prime \prime}=0$, since $E^{\prime}$ is indecomposable and $E^{\prime \prime \prime} \approx E_{2} \neq 0$. Hence $E_{1}=\mathcal{O}_{X}$. This shows that the exact sequence $0 \longrightarrow E^{\prime} \stackrel{\alpha}{\longrightarrow} E$ $\stackrel{\varphi}{\longrightarrow} \mathcal{O}_{X} \longrightarrow 0$ splits. This is a contradiction. Therefore $E$ is indecomposable.

Proof of Proposition 17. When $g=1 . \quad \Omega_{X}^{1} \approx \mathcal{O}_{X}$ and $F_{r}$ has a splitting $\left(\mathcal{O}_{X}, \mathcal{O}_{X}, \cdots, \mathcal{O}_{X}\right)$ (cf. [1]).

When $g>1$. We prove this by induction on $r$.

$r=1$. It is obvious.

$r>1$. By induction assumption, there exists an indecomposable vector $F_{r-1}$ which has a splitting $\left(\Omega_{X}^{1 \otimes(r-2)}, \Omega_{X}^{1 \otimes(r-3)}, \cdots, \Omega_{X}^{1}, \mathcal{O}_{X}\right)$. Since $H^{1}\left(X, F_{r-1} \otimes \Omega_{X}^{1}\right)=H^{0}\left(X, \check{F}_{r-1}\right) \neq 0$, the exists a non-split exact sequence $0 \rightarrow F_{r-1} \otimes \Omega_{X}^{1} \rightarrow E \rightarrow \mathcal{O}_{X} \rightarrow 0$.

Applying Lemma 19 to this exact sequence, we see that $E$ is indecomposable. It is easy to show that $E$ has a splitting $\left(\Omega_{X}^{1 \otimes(r-1)}, \Omega_{X}^{1 \otimes(r-2)}, \ldots, \mathcal{O}_{X}\right)$. 
Proposition 20. Let $X$ be a curve of genus $g \geqq 2$. Let $E$ be an indecomposable vector bundle of rank $r$ on $X$ and let $\left(L_{1}, L_{2}, \cdots, L_{r}\right)$ be a maximal splitting of $E$. If $d_{0}=\min \left\{\operatorname{deg} L_{1}, \operatorname{deg} L_{2}, \cdots, \operatorname{deg} L_{r}\right\}$, then

$$
\operatorname{deg} E \leqq r(r-1)(g-1)+r d_{0} \text {. }
$$

In order to prove Proposition 20, we need the following Lemmas.

Lemma 21. Let $X$ be a curve of genus g. Let $E$ (resp. $\left.E^{\prime}\right)$ be a vector bundle of rank $r$ (resp. $\left.r^{\prime}\right)$ on $X$ and let $\left(\right.$ resp. $\left(M_{1}, M_{2}, \cdots, M_{s}\right)$ ) be a splitting of $E$ (resp. $\left.E^{\prime}\right)$. Suppose that $\operatorname{deg} L_{i}>\operatorname{deg} M_{j}+2(g-1)$, for all $i, j$, then $H^{1}\left(X, \check{E} \otimes E^{\prime}\right)=0$.

Proof of Lemma 21. $\left(L_{i} \otimes \check{M}_{j}\right) i, j$ is a splitting of $E \otimes \check{E}$. Since $\operatorname{deg} \Omega_{X}^{1} \otimes M_{j} \otimes \check{L}_{i}<0$, we have $H^{1}\left(X, L_{i} \otimes \check{M}_{j}\right)=H^{0}\left(X, \Omega_{X}^{1} \otimes M_{j} \otimes \check{L}_{i}\right)=0$. Therefore we have $H^{1}\left(X, E \otimes \check{E}^{\prime}\right)=0$.

LEMMA 22. Let $X$ be a curve of genus $g$. Let $E$ be an indecomposable vector bundle of rank $r$ on $X$ and let $\left(L_{1}, L_{2}, \cdots, L_{r}\right)$ be a splitting of $E$. Then for any $m$ with $1 \leqq m \leqq r$, we have

$$
\begin{aligned}
\min & \left\{\operatorname{deg} L_{1}, \operatorname{deg} L_{2}, \cdots, \operatorname{deg} L_{m-1}\right\} \\
& \leqq \max \left\{\operatorname{deg} L_{m}, \operatorname{deg} L_{m+1}, \cdots, \operatorname{deg} L_{r}\right\}+2(g-1) .
\end{aligned}
$$

Proof of Lemma 22. It is obvious by virtue of Lemma 21.

Lemma 23 (M. Nagata). Let $X$ be a curve of genus $g$. Let $E$ be a vector bundle of rank 2 and let $\left(L_{1}, L_{2}\right)$ be a maximal splitting of $E$. Then

$$
\operatorname{deg} L_{2} \leqq \operatorname{deg} L_{1}+g \text {. }
$$

Proof of Lemma 23. See M. Nagata [7] or M. Maruyama [6] Theorem 3. 13.

LEMMA 24. Let $X$ be a curve of genus $g$. Let $E$ be a vector bundle of rank $r$ on $X$ and let $\left(L_{1}, L_{2}, \cdots, L_{r}\right)$ be a maximal splitting of $E$. Then

$$
\operatorname{deg} L_{r} \leqq \operatorname{deg} L_{1}+(r-1) g .
$$

Proof of Lemma 24. It is obvious by virtue of Lemma 23.

Proof of Proposition 20. We shall define a sequence of integers, 
$1=i_{n}<i_{n-1}<, \cdots,<i_{2}<i_{1}<i_{0}=r+1$, which satisfies the following condition.

$$
\operatorname{deg} L_{i_{m}}=\min \left\{\operatorname{deg} L_{1}, \operatorname{deg} L_{2}, \cdots, \operatorname{deg} L_{i_{m-1}-1}\right\} \quad(m>0) .
$$

We define a one-to-one onto map

$$
\varphi:\{1,2, \cdots, r\} \longrightarrow\{0,1, \cdots, r-1\},
$$

such that $\varphi(j)=r+j-i_{m}-i_{m-1}+1$ where $i_{m} \leqq j<i_{m-1}$. We shall prove that

$$
\operatorname{deg} L_{j} \leqq d_{0}+2 \varphi(j)(g-1)
$$

by induction on $m$ such that $i_{m} \leqq j<i_{m-1}$.

For $m=1$. Since $\left(L_{i_{1}}, L_{i_{1}+1}, \cdots, L_{j}\right)$ is a maximal splitting of a vector bundle, we have $\operatorname{deg} L_{j} \leqq d_{0}+\left(j-i_{1}\right) g$, by virtue of Lemma 24 . Since $\varphi(j)=j-i_{1}$ and $g \leqq 2(g-1)$, we have

$$
\operatorname{deg} L_{j} \leqq d_{0}+2 \varphi(j)(g-1) .
$$

For $m>1$. Since $\left(L_{i_{m}}, L_{i_{m}}+1, \ldots, L_{j}\right)$ is a maximal splitting of a vector bundle, we have $\operatorname{deg} L_{j} \leqq \operatorname{deg} L_{i_{m}}+\left(j-i_{m}\right) g \leqq \operatorname{deg} L_{i_{m}}+2\left(j-i_{m}\right)$ $(g-1)$. Since $\varphi\left(i_{m-2}-1\right) \geqq \varphi(q)$ for all $i_{m-1} \leqq q \leqq r$, we have

$$
\operatorname{deg} L_{q} \leqq d_{0}+2 \varphi(q)(g-1) \leqq d_{0}+2 \varphi\left(i_{m-2}-1\right)(g-1) \text {, }
$$

for all $i_{m-1} \leqq q \leqq r$, by induction assumption. For any $1 \leqq q^{\prime}<i_{m-1}$, $\operatorname{deg} L_{q^{\prime}} \geqq \operatorname{deg} L_{i_{m}}$. Hence by virtue of Lemma 22, we have

$$
\operatorname{deg} L_{i_{m}} \leqq d_{0}+2 \varphi\left(i_{m-2}-1\right)(g-1)+2(g-1) .
$$

Hence we have

$$
\begin{aligned}
\operatorname{deg} L_{j} & \leqq d_{0}+2\left(r-i_{m-1}+1\right)(g-1)+2\left(j-i_{m}\right)(g-1) \\
& =d_{0}=2 \varphi(j)(g-1) .
\end{aligned}
$$

Therefore, we have

$$
\operatorname{deg} E=\sum_{j=1}^{r} \operatorname{deg} L_{j} \leqq r d_{0}+\sum_{j=1}^{r} 2 \varphi(j)(g-1)=r d_{0}+r(r-1)(g-1) .
$$

THEOREM 25. Let $X$ be a curve of genus $g>1$. Then

(i) for any indecomposable vector bundle of rank $r$ such that $\operatorname{deg} E>r(r-1)+(g-1)+r n(X)$, the Frobenius map $F^{*}(1, \check{E})$ is injective. 
(ii) if $\boldsymbol{n}(X)>0$, then for any $r>0$, there exists an indecomposable vector bundle $E^{\prime}$ of rank $r$ with $\operatorname{deg} E^{\prime}=r(r-1)(g-1)+r \boldsymbol{n}(X)$ such that the Frobenius map $F^{*}\left(1, \check{E}^{\prime}\right)$ is not injective

Proof. (i) Let $\left(L_{1}, L_{2}, \cdots, L_{r}\right)$ be a maximal splitting of $E$. Then $\operatorname{deg} L_{j}>\boldsymbol{n}(X)$, by virtue of Proposition 20. Hence the Frobenius map $F^{*}\left(1, L_{j}\right)$ is surjective for all $j$, and the Frobenius map $F^{*}(1, E)$ is surjective. Therefore, the Frobenius map $F^{*}(1, \check{E})$ is injective by virtue of Corollary 8.

(ii) When $\boldsymbol{n}(X)>0$, there exists a line bundle $M$ of degree $\boldsymbol{n}(X)$, such that the Frobenius map $F^{*}(1, \check{M})$ is not injective. There exists an indecomposable vector bundle $F_{r}$ which has a splitting $\left(\Omega_{X}^{1 \otimes(r-1)}, \Omega_{X}^{1 \otimes(r-2)}, \ldots, \Omega_{X}^{1}, \mathcal{O}_{X}\right)$. Put $E^{\prime}=F_{r} \otimes M$. Then $E^{\prime}$ is an indecomposable vector bundle of rank $r$, and of degree $r(r-1)(g-1)+r \boldsymbol{n}(X)$, which has $M$ as a quotient line bundle. And $H^{0}\left(X, \check{E}^{\prime}\right)=H^{0}\left(X, \check{E}^{\prime(p)}\right)=0$. Therefore, the Frobenius map $F^{*}\left(1, \check{E}^{\prime}\right)$ is not injective, by virtue of Corollary 6 .

5. In this section we shall give an example of a curve with positive $\boldsymbol{n}(X)$ although the Hasse-Witt matrix of $X$ is non-singular. We also give other examples of a curve $X$ with positive $n(X)$.

EXAMPLE 1. Let $k$ be an algebraically closed field of characteristic 3. Let $X \subset \boldsymbol{P}_{k}^{2}$ be the curve defined by the homogeneous equation

$$
X_{0}^{3} X_{1}+X_{1}^{3} X_{2}+X_{2}^{3} X_{0}=0 \text {. }
$$

One verifies easily that $X$ is non-singular. Being a plane curve of degree 4, it has genus 3. (This example was given in [5]). The Hasse-Witt matrix of $X$ is identically zero. (cf. [5]).

Proposition 26. If $X$ is the curve in Example 1 , then $n(X)=1$.

Proof. By Definition 11, $\boldsymbol{n}(X) \leqq 1$. Let $f=\left(X_{0}-X_{2} / X_{1}\right) \in K=K(X)$. We have $(f)_{\infty}=(0,0,1)+3(1,0,0)$. This shows that $f \notin K^{3}$. It is easy to show that $v_{x}(\mathrm{~d} f) \geqq-3$, if $\mathrm{x}=(0,0,1)$ or $x=(1,0,0)$, and $v_{x}(\mathrm{~d} f) \geqq 3$, if $x=(1-\alpha,-1,1) \quad i=1,2,3$ where $\alpha_{i}$ are the distinct roots of the equation $\alpha^{3}=\alpha+1$, and $v_{x}(\mathrm{~d} f) \geqq 0$, if $x \neq(1,0,0)$. This shows that $\boldsymbol{n}(f) \geqq 1$, and $\boldsymbol{n}(X)=1$.

EXAMPLE 2. Let $k$ be an algebraically closed field of characteristic 3. Let $X \subset \boldsymbol{P}_{k}^{2}$ be the curve defined by the homogeneous equation 


$$
X_{0}^{4}-X_{1}^{3} X_{2}-X_{1} X_{2}^{3}=0 .
$$

One verifies easily that $X$ is non-singular. Being a plane curve of degree 4, it has genus 3. (This example was given in [2]). The Hasse-Witt matrix of $X$ is identically zero. (cf. [2]).

Proposition 27. If $X$ is the curve in Example 2, theh $n(X)=1$.

Proof. We prove this in the same way as in Proposition 26. We have $\boldsymbol{n}(X) \leqq 1$. Put $f=\left(X_{2} / X_{1}\right) \in K$, then $\boldsymbol{n}(f)=1$. Therefore we have $\boldsymbol{n}(X)=1$.

EXAMPLE 3. Let $k$ be an algebraically closed field of characteristic $p \geqq 3$. Let $X \subset \boldsymbol{P}_{k}^{2}$ be the curve defined by the homogeneous equation

$$
X_{0}^{p+1}=X_{1} X_{2}\left(X_{0}^{p-1}+X_{1}^{p-1}-X_{2}^{p-1}\right) .
$$

One verifies easily that $X$ is non-singular. Being a plane curve of degree $p+1$, it has genus $(1 / 2) p(p-1)$.

Proposition 28. If $X$ is the curve in Example 3, then $n(X)=p$ $-2>0$.

Proof. We have $n(X) \leqq p-2$. Put $f=\left(X_{0} / X_{1}\right) \in K$, then we have $n(X)=p-2$.

Proposition 29. If $X$ is the curve in Example 3, then the HasseWitt matrix is non-singular, i.e., the Frobenius endomorphism of $H^{1}\left(X, \mathcal{O}_{X}\right)$ is injective.

Proof. $U_{i}=\left\{\left(X_{0}, X_{1}, X_{2}\right) ; X_{i} \neq 0\right\} i=1,2$ are affine open subsets of $\boldsymbol{P}_{k}^{2}$. Then $X \subset U_{1} \cup U_{2}$. Let $f=X_{0}^{p+1}-X_{1} X_{2}\left(X_{0}^{p-1}+X_{1}^{p-1}-X_{2}^{p-1}\right) \in$ $k\left[X_{0}, X_{1}, X_{2}\right]$. Now let $\alpha \in H^{1}\left(X, \mathcal{O}_{X}\right)$. Since $\left\{X \cap U_{1}, X \cap U_{2}\right\}$ is an affine open covering of $X$, we can realize $\alpha$ as a function $\bar{h}$ on $X \cap U_{1} \cap U_{2}$. This function extends to a function $h$ on $U_{1} \cap U_{2}$, i.e., to an element of the ring $k\left[X_{0} / X_{1}, X_{2} / X_{1}, X_{1} / X_{2}\right]$. The set of coboundaries is

$$
\left\{h_{1}-h_{2} ; h_{1} \in k\left[\frac{X_{0}}{X_{1}}, \frac{X_{2}}{X_{1}}\right], h_{2} \in k\left[\frac{X_{0}}{X_{2}}, \frac{X_{1}}{X_{2}}\right]\right\}
$$

$h$ is a linear combination of monomials $X_{0}^{i} / X_{1}^{j} X_{2}^{i-j}$. Now if $i \geqq p+1$, we can write 


$$
\frac{X_{0}^{i}}{X_{1}^{j} X_{2}^{i-j}} \equiv \frac{X_{0}^{i-2}}{X_{1}^{j-1} X_{2}^{i-j-1}}+\frac{X_{0}^{i-p-1}}{X_{1}^{j-p} X_{2}^{i-j-1}}+\frac{X_{0}^{i-p-1}}{X_{1}^{j-1} X_{2}^{i-j-p}} \quad(\bmod f)
$$

If $i \leqq j$ or $j \leqq 0$, then $X_{0}^{i} / X_{1}^{j} X_{2}^{i-j}$ is a coboundary. Let $\varphi$ be the natural map $k\left[X_{0} / X_{1}, X_{2} X_{1}, X_{1} X_{2}\right] \rightarrow H^{1}\left(K, \mathcal{O}_{X}\right)$. Then we can choose $\varphi\left(X_{0}^{2} / X_{1} X_{2}\right)$, $\varphi\left(X_{0}^{3} / X_{1} X_{2}^{2}\right), \varphi\left(X_{0}^{3} / X_{1}^{2} X_{2}\right), \cdots, \varphi\left(X_{0}^{p} / X_{1} X_{2}^{p-1}\right), \varphi\left(X_{0}^{p} / X_{1}^{2} X_{2}^{p-2}\right), \cdots, \varphi\left(X_{0}^{p} / X_{1}^{p-1} X_{2}\right)$ as a basis of $H^{1}\left(X, \mathcal{O}_{X}\right)$. Let $\alpha_{\varepsilon i j}=\left(X_{0}^{p-2 j+\varepsilon-1} / X_{1}^{i-j} X_{2}^{p-i-j+\varepsilon-1}\right)$, for all $i, j$ and $\varepsilon=0$ or 1 . To complete the proof we need the following Lemma.

LEMMA 30. Under the same notation as above,

(i) let $V_{s i}$ be a vector subspace of $H^{1}\left(X, \mathcal{O}_{X}\right)$ which is spanned by $\alpha_{\varepsilon i 0}, \alpha_{\varepsilon i 1}, \cdots, \alpha_{\varepsilon i j(\varepsilon i)}$ where $j(\varepsilon i)=\min \{i-1, p-i+\varepsilon-2\}$, for all $i$ such that $p+\varepsilon-2 \geqq i \geqq 1$. Then $V_{\varepsilon i}$ is stable under the Frobenius endomorphism.

(ii) $F^{*}\left(1, \mathcal{O}_{X} \mid V_{\varepsilon i}\right.$ is an injection.

(iii) $\bigoplus_{\varepsilon, i} V_{s i}=H^{1}\left(X, \mathcal{O}_{X}\right)$.

Proof. Let $1 / 2(p-1) \geqq j \geqq 1$, then we have

$$
\begin{aligned}
& X_{0}^{p(p-2 j+\varepsilon+1)}-X_{0}^{p(p-2 j+\varepsilon-1)}\left(X_{1} X_{2}\right)^{p} \\
& =X_{0}^{p(p-2 j+\varepsilon-1)}\left(X_{0}^{2}-X_{1} X_{2}\right)^{p} \\
& \equiv X_{0}^{p-2 j+\varepsilon-1}\left(X_{0}^{2}-X_{1} X_{2}\right)^{2 j-\varepsilon+1} X_{0}^{p-1}\left(X_{0}^{2}-X_{1} X_{2}\right)^{p-2 j+\varepsilon-1} \\
& X_{0}^{p-2 j+\varepsilon-1}\left(X_{0}^{2}-X_{1} X_{2}\right)^{2 j-\varepsilon+1}\left(X_{1} X_{2}\right)^{p-2 j+\varepsilon-1}\left(X_{1}^{p-1}-X_{2}^{p-1}\right)^{p-2 j+\varepsilon-1} \\
& (\bmod f) \\
& =\sum_{m=0}^{j}(-1)^{m}\left(\begin{array}{c}
2 j-\varepsilon+1 \\
m
\end{array}\right) X_{0}^{p-2 j+2 m+\varepsilon-1}\left(X_{1} X_{2}\right)^{p-m}\left(X_{1}^{p-1}-X_{2}^{p-1}\right)^{p-2 j+\varepsilon-1} \\
& +\sum_{m=1}^{j-\varepsilon+1}(-1)^{j+m}\left(\begin{array}{c}
2 j-\varepsilon+1 \\
j+\mathrm{m}
\end{array}\right) X_{0}^{p+2 m+\varepsilon-1}\left(X_{1} X_{2}\right)^{p-m-j}\left(X_{1}^{p-1}-X_{2}^{p-1}\right)^{p-2 j+\varepsilon-1} \\
& \equiv \sum_{m=0}^{j}(-1)^{m}\left(\begin{array}{c}
2 j-\varepsilon+1 \\
m
\end{array}\right) X_{0}^{p-2 j+2 m+\varepsilon-1}\left(X_{1} X_{2}\right)^{p-m}\left(X_{1}^{p-1}-X_{2}^{p-1}\right)^{p-2 j+\varepsilon-1} \\
& +\sum_{m=1}^{j-\varepsilon+1}\left(\sum_{n=m}^{j-\varepsilon+1}(-1)^{j+n}\left(\begin{array}{c}
2 j-\varepsilon+1 \\
j+n
\end{array}\right)\right) X_{0}^{2 m+\varepsilon-2}\left(X_{1} X_{2}\right)^{p-m-j+1} \\
& \times\left(X_{1}^{p-1}-X_{2}^{p-1}\right)^{p-2 j+\varepsilon} \\
& +\left(\sum_{n=1}^{j-\varepsilon+1}(-1)^{j+n}\left(\begin{array}{c}
2 j-\varepsilon+1 \\
j+n
\end{array}\right)\right) X_{0}^{p+\varepsilon-1}\left(X_{1} X_{2}\right)^{p-j}\left(X_{1}^{p}-X_{2}^{p-1}\right)^{p-2 j+\varepsilon-1}
\end{aligned}
$$

In the sequel, let $p>i$ and $0 \leqq j-1 \leqq j(\varepsilon i)$. Then we have 


$$
\begin{gathered}
\varphi\left(\frac{X_{0}^{p-2 j+2 m+\varepsilon-1}\left(X_{1} X_{2}\right)^{p-m}\left(X_{1}^{p-1}-X_{2}^{p-1}\right)^{p-2 j+\varepsilon-1}}{\left(X_{1}^{i-j+1} X_{2}^{p-i-j+\varepsilon}\right)^{p}}\right) \\
=(-1)^{i-j}\left(\begin{array}{c}
p-2 j+\varepsilon-1 \\
i-j
\end{array}\right) \varphi\left(\frac{X_{0}^{p-2 j+2 m+\varepsilon-1}}{X_{1}^{i-j+m} X_{2}^{p-i-j+m+\varepsilon-1}}\right) \\
=(-1)^{i-j}\left(\begin{array}{c}
p-2 j+\varepsilon-1 \\
i-j
\end{array}\right) \alpha_{i j-m} \cdot \\
\varphi\left(\frac{X^{2 m+\varepsilon-2}\left(X_{1} X_{2}\right)^{p-m-j+1}\left(X_{1}^{p-1}-X_{2}^{p-1}\right)^{p-2 j+\varepsilon}}{\left(X_{0}^{i-j+1} X_{2}^{p-i-j+\varepsilon}\right)}\right)=0 .
\end{gathered}
$$

By virtue of formulas (1), (2) and (3), we have

$$
\begin{aligned}
& F^{*}\left(1, \mathcal{O}_{X}\right)\left(\alpha_{s i j-1}\right)-F^{*}\left(1, \mathcal{O}_{X}\right)\left(\alpha_{s i j}\right) \\
& =\sum_{m=0}^{j-1}(-1)^{m}\left(\begin{array}{c}
2 j-\varepsilon+1 \\
m
\end{array}\right)(-1)^{i-j}\left(\begin{array}{c}
p-2 j+\varepsilon-1 \\
i-j
\end{array}\right) \alpha_{\varepsilon i j-m} \\
& +a_{\varepsilon i j-1}(-1)^{i-j}\left(\begin{array}{c}
p-2 j+\varepsilon-1 \\
i-j
\end{array}\right) \alpha_{\varepsilon i 0} \text {, }
\end{aligned}
$$

where $a_{s i j-1}=\sum_{m=0}^{j-\varepsilon+1}(-1)^{j+m}\left(\begin{array}{c}2 j-\varepsilon+1 \\ j+m\end{array}\right)$

Since $j(\varepsilon i)+1 \leqq(1 / 2)(p-1)$ and $\alpha_{\varepsilon i j(\varepsilon i)+1}=0$, formula (4) shows that (i) is true.

(ii) Since $a_{\varepsilon i j-1}+\sum_{m-0}^{j-1}(-1)^{m}\left(\begin{array}{c}2 j-\varepsilon+1 \\ m\end{array}\right)=0$, it is easy to verify that $F^{*}\left(1, \mathcal{O}_{X}\right) \mid V_{i}$ is injective

(iii) It is obvious.

\section{BIBLIOGRAPHY}

[1] M. F. Atiyah, Vector bundle over an elliptic curve. Proc. Lond. Math. Soc. (3) 7 (1957) 414-452.

[2] D. Gieseker, p-ample bundles and their Chern classes. Nagoya Math. J. 43 (1971).

[3] A. Grothendieck and J. Dieudonné, Elément de géométrie algébrique. Publ. Math. I. H. E. S.

[4] R. Hartshorne, Ample vector bundles. Publ. Math. I. H.E. S. 29 (1966).

[ 5 ] R. Hartshorne, Ample vector bundles on curves. Nagoya Math. J. 43 (1971).

[6 ] M. Maruyama, On classification of ruled surfaces. Kinokuniya book store Co. Ltd. Lectures in mathematics Dep. of Math. Kyoto Univ. 3.

[7] M. Nagata, On self intersection number of a section on a ruled surface. Nagoya Math. J. 37 (1970).

[8] T. Oda, Vector bundle on an elliptic curve. Nagoya Math. J. 43 (1971).

[ 9 ] J. P. Serre, Sur la topologié des variété algébraiques en characteristic p. Sympos. Internac. Topologia algebraica. Mexico City. (1956). 24-53. Univ. Nac. Aut. Mexico. 1958. MR 20. 4559. 
[10] P. Cartier, Questions de rationalité des diviseurs en géométrie algébrique. Bull. Soc. Math. France. 86 (1958).

[11] C. S. Seshadri, L'opération de Cartier. Applications. Séminair Chevalley 3 (1958-59) Variétés de Picard.

Department of Mathematics,

Kyoto University 